Article

\title{
Isolates, Antimicrobial Susceptibility Profiles and Multidrug Resistance of Bacteria Cultured from Pig Submissions in New Zealand
}

\author{
Christopher B. Riley ${ }^{1, *(\mathbb{D})}$, Kirsty L. Chidgey ${ }^{2}$, Janis P. Bridges ${ }^{1}$, Emma Gordon ${ }^{1}$ \\ and Kevin E. Lawrence ${ }^{1}$ \\ 1 School of Veterinary Science, Massey University, Palmerston North 4442, New Zealand; \\ bridges@xtra.co.nz (J.P.B.); e.gordon1@massey.ac.nz (E.G.); K.Lawrence@massey.ac.nz (K.E.L.) \\ 2 School of Agriculture and Environment, Massey University, Palmerston North 4442, New Zealand; \\ K.L.Chidgey@massey.ac.nz \\ * Correspondence: c.b.riley@massey.ac.nz
}

Received: 30 July 2020; Accepted: 12 August 2020; Published: 14 August 2020

Simple Summary: Data on the bacterial pathogens and the frequency of antimicrobial resistance (AMR) in New Zealand's pork industry are limited. This study describes bacterial isolates, antimicrobial susceptibility data, and multidrug resistance (MDR; resistance to $\geq 3$ antimicrobial classes) from New Zealand pig submissions. Porcine bacterial culture test results from June 2003 to February 2016 were obtained from commercial veterinary pathology laboratory records. In total, 470/477 unique submissions resulted in bacterial growth, yielding 779 isolates. Sample type was recorded for $75.5 \%$; lung $(21.9 \%)$, faecal $(16.9 \%)$ and intestinal $(12.5 \%)$ were most common. The most common isolates were Escherichia coli (23.9\%), Actinobacillus pleuropneumoniae (5.5\%), Streptococcus suis (5.5\%), unidentified Campylobacter spp. (4.9\%), alpha hemolytic Streptococci (4.1\%), coagulase negative Staphylococcus spp. (3.3\%), and Pasteurella multocida (3.2\%). Susceptibility results were available for 141/779 (18.1\%) isolates from 62/470 (13.2\%) submissions. Most were susceptible to trimethoprim-sulphonamide $(92.6 \%)$, but fewer were susceptible to penicillin $(48.1 \%)$, tilmicosin $(41.9 \%)$, or tetracyclines $(36.0 \%)$. No susceptibility data were for available Salmonella spp., Campylobacter spp., or Yersinia spp. isolates. MDR occurred in $42.6 \%$ of tested isolates. Data on sample submission drivers, antimicrobial drug use, and susceptibilities of important porcine bacterial isolates are required to inform guidelines for prudent antimicrobial use, to reduce their prevalence and MDR.

Abstract: Data on the scope of bacterial pathogens present and the frequency of antimicrobial resistance (AMR) in New Zealand's pigs are limited. This study describes bacterial isolates, antimicrobial susceptibility data, and multidrug resistance (MDR; resistance to $\geq 3$ antimicrobial classes) from New Zealand pig submissions. Porcine test data from June 2003 to February 2016 were obtained from commercial veterinary pathology laboratory records. In total, 470/477 unique submissions resulted in bacterial growth, yielding 779 isolates. Sample type was recorded for 360/477 (75.5\%); lung $(79 / 360 ; 21.9 \%)$, faecal $(61 / 360 ; 16.9 \%)$ and intestinal $(45 / 360 ; 12.5 \%)$ were most common. The most common isolates were Escherichia coli (186/779, 23.9\%), Actinobacillus pleuropneumoniae (43/779; 5.5\%), Streptococcus suis $(43 / 779 ; 5.5 \%)$, unidentified Campylobacter spp. (38/779; $4.9 \%)$, alpha haemolytic Streptococci $(32 / 779 ; 4.1 \%)$, coagulase negative Staphylococcus spp. $(26 / 779 ; 3.3 \%)$, and Pasteurella multocida $(25 / 779 ; 3.2 \%)$. Susceptibility results were available for $141 / 779(18.1 \%)$ isolates from $62 / 470$ (13.2\%) submissions. Most were susceptible to trimethoprim-sulphonamide (75/81; 92.6\%), but fewer were susceptible to penicillin $(37 / 77 ; 48.1 \%)$, tilmicosin $(18 / 43 ; 41.9 \%)$, or tetracyclines $(41 / 114 ; 36.0 \%)$. No susceptibility data were available for Salmonella spp., Campylobacter spp., or Yersinia spp. isolates. MDR was present in 60/141 (42.6\%) isolates. More data on sample submission drivers, antimicrobial drug use, and susceptibilities of important porcine bacterial isolates are required to inform guidelines 
for prudent antimicrobial use, to reduce their prevalence, human transmission, and to minimise AMR and MDR.

Keywords: antimicrobial; resistance; multidrug; bacteria; susceptibility; pig; pork; porcine

\section{Introduction}

Increased recognition of the consequences of antimicrobial resistance (AMR) in humans and animals has led to monitoring and surveillance programs in many countries, often specific to the species being monitored [1]. They range in complexity from highly structured and regulated systems to those that are passive or reactive. Except for a few species of public health interest such as Salmonella spp. [2], current AMR surveillance for bacterial isolates recovered from food animal species in New Zealand falls within the latter approach, relying upon the monitoring of abattoir samples for public health purposes, without direct surveillance of livestock populations. Such surveillance may be more critical within intensive food animal sectors, such as the pig industry, where the use of antibiotics within feedstuffs [3,4] may contribute to levels of antimicrobial resistance in clinically ill and healthy animals [5-7].

The size of the commercial pig population in New Zealand is modest by international standards. It includes approximately 100 commercial farms with an average herd size of 300 sows from which 645,900 offspring were weaned in 2018 [8,9]. There are approximately an additional 7000 small pig holdings including the descendants of a small number of domestic Asiatic pigs introduced in the 19th century, now called the New Zealand Kune Kune [10,11]. Despite the relatively small size of the New Zealand pork industry, data on the pathogenic and opportunistic bacterial species affecting these animals is limited [12-15]. An understanding of the bacterial pathogens and the frequency of AMR of isolates from the national pig population is currently limited in scope and recency $[4,16,17]$.

Veterinarians and their clients may submit samples for bacterial culture and antimicrobial susceptibility testing as part of their herd health management practice, or in response to clinical morbidity and mortality. In New Zealand, some organisms of public health interest are submitted for further evaluation and recording in a centrally managed national database [18]. However, the arising porcine AMR data for most organisms are not. Notwithstanding the limitations of interpreting these data within the context of the national pig population, the systematic collation of data generated from these submissions may contribute to the passive surveillance of AMR in the pork industry [19]. This approach may also be of assistance in underpinning relevant antimicrobial use policies [20], and for understanding the risk that bacterial pathogens and AMR may pose to public health and New Zealand's pork industry. The purpose of this study is to describe bacterial isolates cultured from porcine laboratory submissions, and proportions of cultured bacterial isolates identified as expressing antimicrobial and multidrug resistance in New Zealand.

\section{Materials and Methods}

Results from the bacterial culture of porcine sample submissions from June 2003 to February 2016 and antimicrobial susceptibility test data for isolates were obtained from five commercial veterinary pathology laboratories located in Auckland, Hamilton, Palmerston North, Christchurch, and Dunedin (Gribbles Veterinary, Healthscope (C), Australia and New Zealand). Data obtained included the submission date, accession number, the signalment of the pig from which the sample was collected, the region within New Zealand from where the sample originated, a specimen description, the bacterial isolates cultured and, if performed, the antimicrobial panel susceptibilities of the isolates. Antimicrobial susceptibility testing was performed using disk diffusion assays [21]. Data identifying the owners or farms from which samples were submitted collected were not available.

Bacterial culture and susceptibility results of all submissions with the sampled species identified as a pig or listed as a porcine breed were evaluated irrespective of age or sex. Data not confidently classified as 
porcine-related were excluded. Specimen descriptions for the samples, stating either the sample type or the anatomical origin were inconsistent. Therefore, they were grouped into broader categories based on anatomical regions or organ systems (e.g., gut and small intestine were grouped within intestinal).

The number of each bacterial species isolated was determined where species data was available, or by genus when the species was not determined. For each species (or genus) the number and proportion of isolates tested for antimicrobial susceptibility were also determined. To reflect changes in nomenclature during the time spanned by the data, organisms grouped as Trueperella pyogenes included those listed as Actinomyces pyogenes or Arcanobacterium pyogenes. Bacteria listed as Actinobacillus pleuropneumoniae included those recorded as Haemophilus pleuropneumoniae.

Organisms recorded as demonstrating marginal or intermediate susceptibility, or as resistant to a tested antimicrobial, were classed as resistant (i.e., not susceptible). An isolate not susceptible to an antimicrobial agent was considered as having AMR to that compound. Bacterial isolates reported as resistant to one agent in three or more antimicrobial classes were identified as multidrug resistant (MDR) [22]. Antimicrobials and their classes identified for determining AMR and MDR respectively included $\beta$-lactams (oxacillin, penicillin and cephalosporins (cephalexin, cephalothin, ceftazidime and ceftiofur)), aminoglycosides (apramycin, gentamicin, neomycin, streptomycin, and spectinomycin), fluoroquinolones (enrofloxacin and marbofloxacin); lincosamides (clindamycin and lincomycin), and macrolides (erythromycin, tilmicosin and tylosin). Antimicrobials that had only a single drug tested within their class included chloramphenicol, fusidic acid, polymixin B, tetracycline, and trimethoprim-sulphonamide.

Summative and comparative analyses were undertaken in Excel (Microsoft Excel ${ }^{\circledR}$, Version 16.21.19012303, Microsoft Corporation, Redmond, WA, USA). Ages of the animals were described numerically for some pigs and categorically for others. For the latter ages were transformed to days, and the median and interquartile range calculated.

\section{Results}

After data screening as described, there were 477 unique porcine laboratory submissions for bacterial culture between June 2003 to February 2016. Of these, 470/477 (98.5\%) yielded bacterial growth producing 779 isolates. Of these, $62 / 470(13.2 \%)$ submissions producing 144 isolates had antimicrobial susceptibility testing performed.

\subsection{Signalment and Submission Data}

Signalment data within the databases was inconsistently described in the databases. Breeds described in descending order of frequency were Large White $(n=59 / 477 ; 12.4 \%)$ or Large White cross $(n=8 / 477 ; 1.7 \%)$, Auckland Island $(n=44 / 477 ; 9.2 \%)$ or Mixed breed $(n=7 / 477 ; 1.5 \%)$, Kune Kune $(n=5 / 477 ; 1.0 \%)$, Landrace $(n=2 / 477 ; 0.4 \%)$ or Landrace cross $(n=3 / 477 ; 0.6 \%)$, Duroc $(n=2 / 477$; $0.4 \%)$, Berkshire $(n=1 / 477 ; 0.2 \%)$ or Saddleback $(n=1 / 477 ; 0.2 \%)$. Breed was not recorded or unknown for $345 / 477(72.3 \%)$ submissions. Records described sex as female $(n=72 / 477 ; 15.1 \%)$, male $(n=66 / 477$; $13.8 \%$ ), mixed ( $n=43 / 477 ; 9 \%)$, or unknown or not recorded $(n=296 / 477 ; 62.1 \%)$. Submission ages were listed either in days $(n=46 / 477)$, weeks $(n=104 / 477)$, months $(35 / 477)$, years $(n=29 / 477)$, or categorically ( $n=263 / 477$ ). The ages of pigs that were listed numerically $(214 / 477 ; 44.9 \%)$ has a median age of 56 days (interquartile range 90 days; min 0 days; max 11 years). The remaining records described the age of the pig as foetus $(n=2 / 477 ; 0.4 \%)$, neonate $(n=4 / 477 ; 0.8 \%)$, young $(n=12 / 477$; $2.5 \%)$, adult or mature $(n=6 / 477 ; 1.3 \%)$, mixed $(n=25 / 477 ; 5.2 \%)$, or unknown $(n=214 / 477 ; 44.9 \%)$. The animal's age was not described (unknown) for 214/477 (44.9\%) records.

Most submissions were received by the Christchurch laboratory $(269 / 477 ; 50.4 \%)$, followed by Palmerston North (93/477; 19.5\%), Auckland (86/477; 18.0\%), Dunedin (24/477; 5.0\%), and Hamilton (5/477; $1 \%$ ) (Figure 1). In decreasing order the number of samples submitted by region were from Canterbury (146/477; 30.6\%), Otago (108/477; 22.6\%), Auckland (70/477; 14.7\%), Manawatu-Wanganui (53/477; 11.1\%), Taranaki (20/477; 4.2\%), Southland (19/477; 4.0\%), Hawke's Bay (15/477; 3.1\%), Marlborough (13/477; 2.7\%), 
Northland (13/477; 2.7\%), Waikato (6/477; 1.3\%), Wellington (5/477; 1.0\%), Gisborne (5/477; $1.0 \%)$, Bay of Plenty $(1 / 477 ; 0.2 \%)$, and the West Coast $(1 / 477 ; 0.2 \%)$. The region from which the sample was submitted was not recorded for $2 / 477(0.4 \%)$ samples.

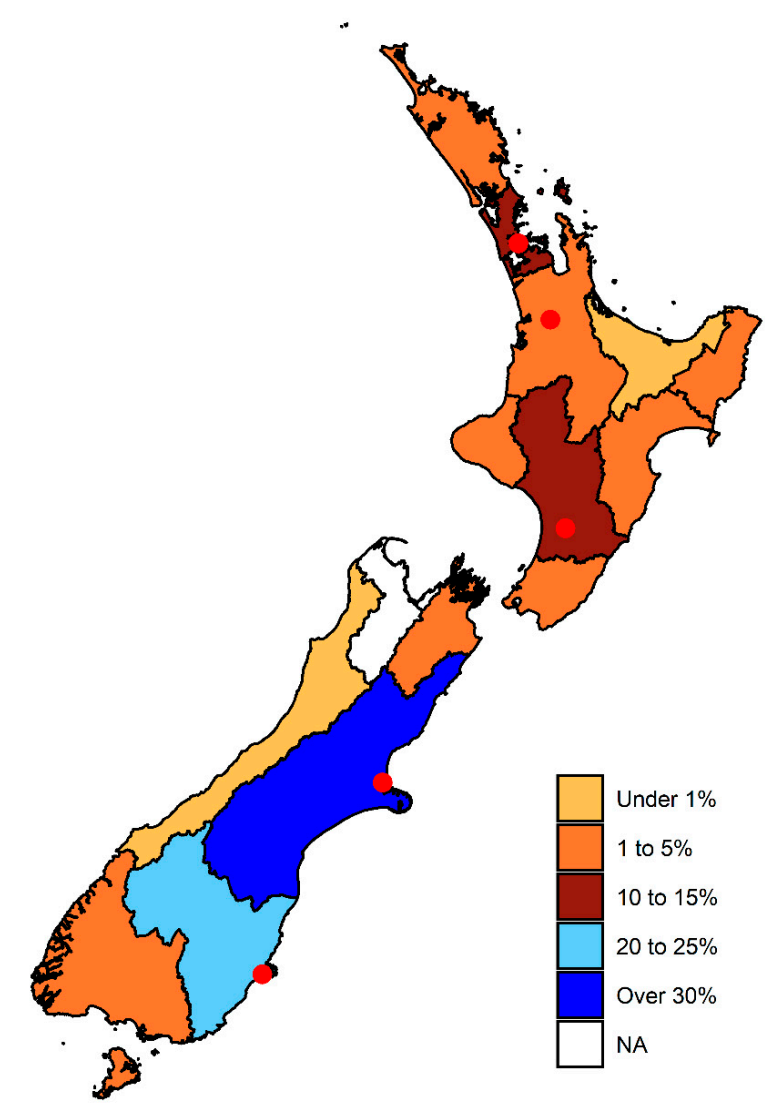

Figure 1. Percentage of submissions $(n=475)$ submitted for bacterial culture and antimicrobial susceptibility testing to commercial veterinary diagnostic laboratories between 2003 and 2016, from pigs in different regions of New Zealand as demarcated on the map. The locations of veterinary laboratories where porcine samples were submitted are indicated as red circles.

Only 360/477 (75.5\%) of submissions recorded a sample type or anatomic origin. The most common submitted specimens were lung (79/360; 21.9\%), faecal (61/360; 16.9\%), intestinal (45/360; 12.5\%), liver (13/360, $3.6 \%)$, stomach $(11 / 360 ; 3.1 \%)$ and semen $(11 / 360 ; 3.1 \%)$. Most submissions were from a single anatomic location or specimen $(315 / 360 ; 87.5 \%), 8 / 360(2.2 \%)$ from multiple gastrointestinal sites, and 37/360 (10.3\%) were from multiple sites or organs (range 2 to 6 sites or tissues). There were $64 / 360(17.8 \%)$ swabs and 25/360 $(6.9 \%)$ tissues submitted without an identified source or origin. Sample sites for the seven most common bacterial isolates cultured from porcine samples for the period 2003 to 2016 are listed in Table 1.

\subsection{Culture Results from Submissions}

The numbers of isolates from submissions identified by species ( $n=57$ species including staphylococcal and streptococcal subtypes $(n=7))$ and those characterised to the genus level $(n=24$ genera) are listed in Table 2. Including staphylococcal and streptococcal subtypes, 539/779 (69.2\%) isolates were identified by species, $139 / 779$ (17.8\%) by genus only, and the remainder (101/779; 13\%) were not identified by species or genus (e.g., mixed bacteria; coliforms; mixed normal flora; etc.) (Table 2). The most commonly identified isolates were Escherichia coli (186/779, 23.9\% for all strains), followed in order of decreasing frequency by Actinobacillus pleuropneumoniae, Streptococcus suis, other Campylobacter spp., alpha hemolytic Streptococci, coagulase negative Staphylococcus spp. and Pasteurella multocida (Table 2). Salmonella spp. (12/779; 1.5\%), C. coli and C. jejuni (29/779:3.7\%), and Yersinia spp. (9/779; $1.2 \%)$ were infrequently isolated. 
Table 1. Sample sites for the seven most common bacterial isolates cultured from porcine samples. The percentage of total isolates for each isolate type by sampling site are shown in parentheses.

\begin{tabular}{|c|c|c|c|c|c|c|c|c|}
\hline \multirow[b]{2}{*}{ Sample Type ${ }^{a}$} & \multicolumn{8}{|c|}{ Species of Bacterial Isolates } \\
\hline & Escherichia coli & $\begin{array}{l}\text { Actinobacillus } \\
\text { pleuropneumoniae }\end{array}$ & $\begin{array}{c}\text { Campylobacter } \\
\text { species }\end{array}$ & $\begin{array}{c}\text { Pasteurella } \\
\text { multocida }\end{array}$ & $\begin{array}{l}\text { Stretocoocus } \\
\text { suis }\end{array}$ & $\begin{array}{c}\text { Staphylococci } \\
\text { Coagulase-Negative }\end{array}$ & $\begin{array}{l}\text { Streptococci alpha } \\
\text { Haemolytic }\end{array}$ & $\begin{array}{l}\text { Total for all } \\
\text { Isolates }\end{array}$ \\
\hline Eye swab & 1/98 (1.0) & - & - & - & - & - & - & $1 / 360(0.3)$ \\
\hline Upper respiratory & - & - & - & - & - & - & - & $3 / 360(0.8)$ \\
\hline Lung & 10/98 (10.2) & $29 / 35(82.9)$ & - & $8 / 15(53.3)$ & $4 / 28(14.3)$ & $1 / 4(25)$ & - & $79 / 360(21.9)$ \\
\hline Heart or pericardial fluid & - & - & - & - & $1 / 28(3.6)$ & - & - & $5 / 360(1.4)$ \\
\hline Abdominal fluid & - & - & - & - & $2 / 28(7.1)$ & - & - & $1 / 360(0.3)$ \\
\hline Stomach & 4/98 (4.1) & - & - & - & - & - & $1 / 6(16.7)$ & $11 / 360$ \\
\hline Intestinal & 22/98 (22.4) & - & $9 / 26(34.6)$ & - & $1 / 28(3.6)$ & - & - & $45 / 360(12.5)$ \\
\hline Colon, caecum or rectal & $2 / 98(2.0)$ & - & $2 / 26(7.7)$ & - & - & - & - & $7 / 360(1.9)$ \\
\hline Faecal & 30/98 (30.6) & - & $14 / 26(53.8)$ & - & - & - & $1 / 6(16.7)$ & $61 / 360(16.9)$ \\
\hline Liver & 1/98 (1.0) & - & - & $1 / 15(6.7)$ & $1 / 28(3.6)$ & - & $1 / 6(16.7)$ & $13 / 360(3.6)$ \\
\hline Spleen & - & - & - & - & $2 / 28(7.1)$ & $1 / 4(25)$ & - & $4 / 360(1.1)$ \\
\hline Kidney & - & - & - & - & $1 / 28(3.6)$ & - & - & $3 / 360(0.8)$ \\
\hline Lymph node & $1 / 98(1.0)$ & - & - & - & - & - & - & $3 / 360(0.8)$ \\
\hline Female urogenital & - & - & - & - & - & - & - & 6/360 (1.7) \\
\hline Semen & 3/98 (3.1) & - & - & - & - & - & - & $11 / 360(3.1)$ \\
\hline Urine & 2/98 (2.0) & - & - & - & - & - & - & $3 / 360(0.8)$ \\
\hline Skin & 1/98 (1.0) & - & - & - & $1 / 28(3.6)$ & - & - & $4 / 360(1.1)$ \\
\hline Foot & - & - & - & - & - & - & - & $2 / 360(0.6)$ \\
\hline Tissue & 3/98 (3.1) & $1 / 35(2.9)$ & $1 / 26(3.8)$ & 3/15 (20.0) & $8 / 28(28.6)$ & $1 / 4(25)$ & - & $25 / 360(6.9)$ \\
\hline Swab & 18/98 (18.4) & $5 / 35(14.3)$ & - & 3/15 (20.0) & $7 / 28(25.0)$ & $1 / 4(25)$ & 2/6 (33.3) & $64 / 360(17.8)$ \\
\hline Abscess & - & - & - & - & - & - & - & $4 / 360(1.1)$ \\
\hline Fluid & - & - & - & - & - & - & - & $1 / 360(0.3)$ \\
\hline Milk & - & - & - & - & - & - & - & $1 / 360(0.3)$ \\
\hline Aspirate & - & - & - & - & - & - & $1 / 6(16.7)$ & $1 / 360(0.3)$ \\
\hline Blood culture & - & - & - & - & - & - & - & $1 / 360(0.3)$ \\
\hline Transport media & - & - & - & - & - & - & - & $1 / 360(0.3)$ \\
\hline
\end{tabular}

a Sample type or site was recorded for only $360 / 477(75.5 \%)$ submissions. 
Table 2. Bacterial species cultured from New Zealand porcine samples submissions and proportion tested for antimicrobial susceptibility.

\begin{tabular}{|c|c|c|c|c|}
\hline \multirow{2}{*}{$\begin{array}{c}\text { Bacterial Species } \\
\text { Acinetobacter johnsonii }\end{array}$} & \multicolumn{2}{|c|}{$\begin{array}{c}\text { Number of } \\
\text { IsolatesCultured }(\%)^{a}\end{array}$} & \multicolumn{2}{|c|}{$\begin{array}{c}\text { Proportion Tested forAntimicrobial } \\
\text { Susceptibility }{ }^{b}(\%)\end{array}$} \\
\hline & 1 & $(0.1)$ & 0 & (0) \\
\hline Actinobacter lwoffii & 2 & $(0.3)$ & $2 / 2$ & (50) \\
\hline Actinobacter species & 9 & (1.2) & $5 / 9$ & $(44.4)$ \\
\hline Actinobacillus pleuropneumoniae & 43 & (5.5) & $2 / 43$ & $(4.7)$ \\
\hline Actinobacillus species & 4 & $(0.5)$ & 0 & (0) \\
\hline Aeromonas species & 2 & $(0.3)$ & 0 & (0) \\
\hline Aeromonas veronii & 1 & $(0.1)$ & 0 & (0) \\
\hline Alcaligenes species & 1 & $(0.1)$ & 0 & (0) \\
\hline Arcanobacterium haemolyticum & 1 & $(0.1)$ & 0 & (0) \\
\hline Arcanobacterium species & 1 & $(0.1)$ & 0 & (0) \\
\hline Bacillus species & 3 & $(0.4)$ & $1 / 3$ & $(33.3)$ \\
\hline Bacteroides species & 4 & $(0.5)$ & $1 / 4$ & $(25)$ \\
\hline Bordetella bronchiseptica & 2 & $(0.3)$ & 0 & $(0)$ \\
\hline Brachyspira (Serpulina) hyodysenteriae & 1 & $(0.1)$ & 0 & (0) \\
\hline Brachyspira pilosicoli & 2 & $(0.3)$ & 0 & (0) \\
\hline Burkholderia cepacian & 4 & $(0.5)$ & $3 / 4$ & (50) \\
\hline Campylobacter coli & 8 & $(1.0)$ & 0 & (0) \\
\hline Campylobacter jejuni & 14 & $(1.8)$ & 0 & (0) \\
\hline Campylobacter jejuni/coli & 7 & $(0.9)$ & 0 & (0) \\
\hline Campylobacter species & 38 & $(4.9)$ & 0 & (0) \\
\hline Campylobacter upsaliensis/helveticus & 1 & $(0.1)$ & 0 & (0) \\
\hline Citrobacter freundii & 1 & $(0.1)$ & 0 & $(0)$ \\
\hline Citrobacter species & 2 & $(0.3)$ & $1 / 2$ & $(50)$ \\
\hline Clostridium perfringens & 7 & $(0.9)$ & 0 & (0) \\
\hline Corynebacterium species & 14 & $(1.8)$ & $4 / 14$ & $(28.6)$ \\
\hline Enterobacter species & 1 & $(0.1)$ & 0 & $(0)$ \\
\hline Enterococcus faecalis & 11 & $(1.4)$ & $5 / 11$ & $(45.5)$ \\
\hline Enterococcus species & 11 & (1.4) & $1 / 11$ & $(9.1)$ \\
\hline Erysipelothrix rhusiopathiae & 1 & $(0.1)$ & 0 & $(0)$ \\
\hline Escherichia coli & 107 & $(13.7)$ & $26 / 107$ & $(24.3)$ \\
\hline Escherichia coli (K88 negative) & 20 & $(2.6)$ & $2 / 20$ & $(10)$ \\
\hline Escherichia coli (K88 positive) & 23 & (3.0) & 0 & $(0)$ \\
\hline Escherichia coli - beta haemolytic & 32 & $(4.1)$ & $4 / 32$ & $(12.5)$ \\
\hline Escherichia coli - beta haemolytic (K88 negative) & 3 & $(0.4)$ & 0 & $(0)$ \\
\hline Escherichia coli - beta haemolytic (K88 positive) & 1 & $(0.1)$ & 0 & (0) \\
\hline Fusobacterium species & 1 & $(0.1)$ & 0 & $(0)$ \\
\hline Haemophilus parasuis & 3 & $(0.4)$ & $1 / 3$ & $(33.3)$ \\
\hline Klebsiella oxytoca & 2 & $(0.3)$ & $2 / 2$ & $(100)$ \\
\hline Klebsiella pneumoniae & 4 & $(0.5)$ & $3 / 4$ & (75) \\
\hline Klebsiella species & 2 & $(0.3)$ & 0 & (0) \\
\hline Lactobacillus species & 1 & $(0.1)$ & 0 & (0) \\
\hline Micrococcus species & 2 & $(0.3)$ & $2 / 2$ & $(100)$ \\
\hline Moraxella species & 1 & $(0.1)$ & 0 & (0) \\
\hline Morganella morganii & 1 & $(0.1)$ & $1 / 1$ & $(100)$ \\
\hline Mycoplasma species & 1 & $(0.1)$ & 0 & $(0)$ \\
\hline Pasteurella multocida & 25 & (3.2) & $4 / 25$ & (16) \\
\hline Pasteurella pneumotropica & 2 & $(0.3)$ & $1 / 2$ & (50) \\
\hline Pasteurella species & 3 & $(0.4)$ & $1 / 3$ & $(33.3)$ \\
\hline Proteus mirabilis & 8 & $(1.0)$ & $3 / 8$ & $(37.5)$ \\
\hline Proteus species & 9 & $(1.2)$ & 0 & $(0)$ \\
\hline Proteus vulgaris & 1 & $(0.1)$ & 0 & $(0)$ \\
\hline Providencia stuartii & 2 & $(0.3)$ & $1 / 2$ & (50) \\
\hline Pseudomonas aeruginosa & 8 & $(1.0)$ & $4 / 8$ & (50) \\
\hline Pseudomonas fluorescens & 1 & $(0.1)$ & 0 & (0) \\
\hline Pseudomonas species & 8 & $(1.0)$ & $2 / 8$ & (25) \\
\hline
\end{tabular}


Table 2. Cont.

\begin{tabular}{|c|c|c|c|c|}
\hline \multirow{2}{*}{$\begin{array}{c}\text { Bacterial Species } \\
\text { Salmonella Brandenburg }\end{array}$} & \multicolumn{2}{|c|}{$\begin{array}{c}\text { Number of } \\
\text { IsolatesCultured (\%) }\end{array}$} & \multicolumn{2}{|c|}{$\begin{array}{l}\text { Proportion Tested forAntimicrobial } \\
\text { Susceptibility }{ }^{b}(\%)\end{array}$} \\
\hline & 1 & $(0.1)$ & 0 & (0) \\
\hline Salmonella species & 4 & $(0.5)$ & 0 & (0) \\
\hline Salmonella Tennessee & 1 & $(0.1)$ & 0 & (0) \\
\hline Salmonella Typhimurium & 6 & $(0.8)$ & 0 & (0) \\
\hline Serratia marcescens & 2 & $(0.3)$ & $1 / 2$ & (50) \\
\hline Staphylococcus aureus & 11 & (1.4) & $4 / 11$ & $(36.4)$ \\
\hline Staphylococcus hyicus & 10 & (1.3) & $3 / 10$ & $(30)$ \\
\hline Staphylococcus intermedius & 3 & $(0.4)$ & 0 & $(0)$ \\
\hline Staphylococcus species & 8 & $(0.1)$ & $1 / 8$ & $(12.5)$ \\
\hline Staphylococcus species - coagulase negative & 26 & (3.3) & $14 / 26$ & $(53.8)$ \\
\hline Streptococcus bovis & 1 & $(0.1)$ & $1 / 1$ & $(100)$ \\
\hline Streptococcus dysgalactiae & 5 & $(0.6)$ & $3 / 5$ & $(60)$ \\
\hline Streptococcus equi subsp. zooepidemicus & 2 & $(0.3)$ & 0 & (0) \\
\hline Streptococcus porcinus & 2 & $(0.3)$ & $\frac{1}{2}$ & $(50)$ \\
\hline Streptococcus suis ${ }^{\mathrm{c}}$ & 43 & (5.5) & $4 / 43$ & (9.3) \\
\hline Streptococcus viridans & 1 & $(0.1)$ & $1 / 1$ & $(100)$ \\
\hline Streptococcus Lancefield Group A & 1 & $(0.1)$ & 0 & $(0)$ \\
\hline Streptococcus Lancefield Group C & 10 & (1.3) & $5 / 10$ & (50) \\
\hline Streptococcus Lancefield Group D & 3 & $(0.4)$ & $2 / 3$ & $(66.7)$ \\
\hline Streptococcus species & 8 & $(1.0)$ & $3 / 8$ & $(37.5)$ \\
\hline Streptococci-alpha haemolytic & 32 & $(4.1)$ & $6 / 32$ & $(18.8)$ \\
\hline Streptococci-beta haemolytic & 10 & (1.3) & $3 / 10$ & $(30)$ \\
\hline Streptococci-non-haemolytic & 3 & $(0.4)$ & $1 / 3$ & $(33.3)$ \\
\hline Trueperella pyogenes & 8 & $(1.0)$ & $1 / 8$ & $(12.5)$ \\
\hline Yersinia enterocolitica & 1 & $(0.1)$ & 0 & $(0)$ \\
\hline Yersinia pseudotuberculosis & 7 & $(0.9)$ & 0 & (0) \\
\hline Yersina species & 1 & $(0.1)$ & 0 & (0) \\
\hline Other not identified to a genus or species & 101 & $(13.0)$ & $8 / 101$ & $(7.9)$ \\
\hline Total susceptibility tested & 779 & $(100)$ & $144 / 779$ & $(18.5)^{d}$ \\
\hline
\end{tabular}

a 779 isolates from 470 submissions; ${ }^{\mathrm{b}} 144$ isolates from 62 submissions; $^{\mathrm{c}}$ One isolate was type 1 , one was type 2 , and the remainder were not subtyped; ${ }^{\mathrm{d}}$ Three organisms $(3 / 144 ; 3 / 779)$ that were submitted for susceptibility testing did not have interpretable results in the record.

\subsection{Antimicrobial Susceptibility and Multidrug Resistance}

The number of antimicrobials each isolate was tested against ranged from 2 to 11 (median 7). For submissions where antimicrobial susceptibility testing was performed, the median number of isolates tested per sample was 2 (range 1-9). Interpretable susceptibility results were available for $141 / 779(18.1 \%)$ isolates from 62/470 (13.2\%) submissions (Table 2). Three organisms (3/144; 3/779) that were submitted for susceptibility testing did not have interpretable results in the database (results were recorded as " $\sim$ DEL"). No antimicrobial susceptibility data were available for isolates of Salmonella spp., Campylobacter spp., or Yersinia spp. Overall, most tested isolates were susceptible to trimethoprim-sulphonamide $(75 / 81 ; 92.6 \%)$, but fewer isolates were susceptible to penicillin (37/77; $48.1 \%)$, tilmicosin $(18 / 43 ; 41.9 \%)$, or tetracyclines $(41 / 114 ; 36.0 \%)$.

There were 60/141 (42.6\%) isolates tested for antimicrobial susceptibility that demonstrated MDR (i.e., resistance to $\geq 3$ antimicrobial classes) (Table 3). Sample sizes within bacterial species were small, but where the same species of the organism was cultured from four or more submissions, MDR was a more frequent finding for Streptococcus suis, alpha-haemolytic and beta-haemolytic Streptococci, and Corynebacterium spp. 
Table 3. Antimicrobial susceptibility and multidrug resistance for tested bacteria isolated from submissions for New Zealand pigs for the period June 2003 to February 2016.

\begin{tabular}{|c|c|c|c|c|c|c|c|c|c|c|c|c|c|c|c|c|c|c|c|c|c|c|c|}
\hline \multirow[b]{2}{*}{ Bacterial Species } & \multicolumn{22}{|c|}{ Antimicrobial Used For Testing Susceptibility ${ }^{a, b}$} & \multirow[b]{2}{*}{ MDR (\%) } \\
\hline & $\sum_{u}^{x}$ & $\sum_{4}^{U}$ & 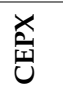 & $\sum_{\vec{U}}^{\mathbb{T}}$ & 预 & گ্ৰ & 莹 & $\frac{\pi}{4}$ & 壳 & $\begin{array}{l}0 \\
\text { Z } \\
\text { Z }\end{array}$ & $\begin{array}{l}u \\
\dot{\omega} \\
\tilde{\omega}\end{array}$ & $\stackrel{\mathscr{G}}{\mathfrak{G}}$ & $\underset{\mathbf{Z}}{\tilde{Z}}$ & $\underset{\Sigma}{\Sigma}$ & 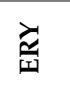 & $\exists$ & $\vec{U}$ & $\underset{u}{U}$ & $\stackrel{-1}{1}$ & $\sum_{i}^{\infty}$ & 焉 & خ্ট & \\
\hline Actinobacter lwoffii & - & $1 / 2$ & $1 / 2$ & - & $1 / 2$ & - & $1 / 2$ & - & $1 / 2$ & $1 / 2$ & - & $1 / 2$ & $0 / 1$ & - & - & - & - & 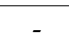 & - & - & - & - & $0 / 2(0)$ \\
\hline Actinobacter specie & $2 / 3$ & $0 / 2$ & - & $2 / 3$ & $2 / 4$ & - & $0 / 3$ & $2 / 3$ & $4 / 5$ & $0 / 3$ & $2 / 2$ & $3 / 4$ & $3 / 4$ & - & - & $1 / 1$ & $0 / 2$ & $0 / 1$ & $3 / 4$ & $3 / 3$ & - & $1 / 1$ & $1 / 6(16.7)$ \\
\hline Actinobacillus pleuropneumoniae & $2 / 2$ & - & - & $2 / 2$ & $2 / 2$ & - & $2 / 2$ & - & - & - & $1 / 1$ & - & $1 / 1$ & - & $1 / 1$ & $1 / 1$ & $2 / 2$ & - & $2 / 2$ & $2 / 2$ & - & - & $0 / 2(0)$ \\
\hline Bacillus species & $1 / 1$ & - & - & - & - & - & $1 / 1$ & - & - & - & - & $0 / 1$ & $0 / 1$ & - & - & - & - & - & $0 / 1$ & $1 / 1$ & - & - & $1 / 1(100)$ \\
\hline Bacteroides species & - & $1 / 1$ & - & $1 / 1$ & $1 / 1$ & - & - & $1 / 1$ & - & $1 / 1$ & - & - & - & - & - & - & - & - & $0 / 1$ & $1 / 1$ & - & - & $0 / 1(0)$ \\
\hline Burkholderia cepacia & $1 / 2$ & $2 / 3$ & - & - & $1 / 1$ & - & $0 / 2$ & $0 / 1$ & $1 / 2$ & $2 / 3$ & $0 / 1$ & $1 / 1$ & $1 / 2$ & - & - & $0 / 2$ & $0 / 1$ & - & $1 / 2$ & - & $0 / 2$ & $1 / 1$ & $2 / 3(66.7)$ \\
\hline Citrobacter species & $0 / 1$ & $0 / 1$ & - & - & - & - & - & - & - & - & - & $0 / 1$ & $1 / 1$ & - & - & - & - & - & $1 / 1$ & - & $1 / 1$ & - & $0 / 1(0)$ \\
\hline Corynebacterium species & $1 / 1$ & $4 / 5$ & $1 / 1$ & - & $2 / 2$ & - & $2 / 4$ & $1 / 3$ & $3 / 4$ & $4 / 5$ & $1 / 2$ & $1 / 1$ & $3 / 4$ & - & $0 / 1$ & $0 / 1$ & $0 / 2$ & $0 / 1$ & $0 / 3$ & - & $0 / 2$ & $2 / 2$ & $3 / 4(75)$ \\
\hline Enterococcus faecalis & $2 / 3$ & $4 / 5$ & - & $1 / 3$ & $2 / 2$ & $0 / 1$ & $4 / 5$ & $0 / 1$ & $0 / 1$ & $0 / 1$ & $1 / 2$ & . & $1 / 2$ & $1 / 1$ & $1 / 2$ & $1 / 4$ & $0 / 3$ & $1 / 1$ & $0 / 5$ & $2 / 3$ & $0 / 1$ & $1 / 1$ & $3 / 5(60)$ \\
\hline Enterococcus species & $0 / 1$ & $0 / 1$ & - & - & - & - & - & - & - & - & - & $0 / 1$ & $1 / 1$ & - & - & - & - & - & $0 / 1$ & $1 / 1$ & - & - & 1/1 (100) \\
\hline Escherichia coli & $9 / 10$ & $7 / 18$ & - & $4 / 5$ & $2 / 3$ & - & $2 / 7$ & $4 / 5$ & $3 / 4$ & $8 / 8$ & $4 / 9$ & $6 / 9$ & $8 / 9$ & - & $3 / 6$ & $2 / 7$ & $1 / 7$ & $1 / 4$ & $6 / 18$ & $13 / 14$ & $40 / 1$ & $2 / 2$ & $9 / 26(34.6)$ \\
\hline Escherichia coli - beta haemolytic & $3 / 3$ & $3 / 4$ & - & - & - & - & - & $1 / 1$ & - & $1 / 2$ & $0 / 1$ & $2 / 2$ & $3 / 3$ & - & - & - & - & - & $0 / 4$ & $4 / 4$ & - & - & $1 / 4(25)$ \\
\hline Escherichia coli (K88 negative) & - & $0 / 1$ & - & $1 / 1$ & - & - & - & $1 / 1$ & - & $2 / 2$ & - & - & $1 / 1$ & - & - & - & - & - & $0 / 2$ & $1 / 2$ & - & - & $1 / 2(50)$ \\
\hline Haemophilus parasuis & $1 / 1$ & - & - & - & - & - & $1 / 1$ & - & - & - & - & $1 / 1$ & $1 / 1$ & - & - & - & - & - & $1 / 1$ & $1 / 1$ & - & - & $0 / 1(0)$ \\
\hline Klebsiella oxytoca & $1 / 1$ & $0 / 1$ & - & - & - & - & $0 / 1$ & $0 / 1$ & $0 / 1$ & $0 / 1$ & $0 / 1$ & - & $1 / 1$ & - & - & $0 / 1$ & $0 / 1$ & - & $0 / 1$ & - & $0 / 1$ & $1 / 1$ & $2 / 2(100)$ \\
\hline Klebsiella pneumoniae & $1 / 1$ & $2 / 4$ & - & - & $1 / 1$ & - & $0 / 1$ & $1 / 1$ & $2 / 2$ & $2 / 2$ & - & $3 / 3$ & - & - & $1 / 2$ & $1 / 1$ & $0 / 2$ & - & $1 / 3$ & $1 / 1$ & - & - & $1 / 3(33.3)$ \\
\hline Micrococcus species & $1 / 1$ & - & - & - & $1 / 1$ & - & - & $1 / 1$ & $1 / 1$ & $1 / 1$ & - & $0 / 1$ & - & - & - & $0 / 1$ & - & - & - & - & - & - & $0 / 1(0)$ \\
\hline Morganella morganii & - & $1 / 1$ & - & - & - & - & $0 / 1$ & - & $1 / 1$ & - & - & $0 / 1$ & $1 / 1$ & - & - & - & - & $0 / 1$ & $1 / 1$ & $1 / 1$ & - & - & $1 / 1(100)$ \\
\hline Pasteurella multocida & $2 / 2$ & $4 / 4$ & - & - & - & - & $2 / 2$ & - & - & - & $1 / 1$ & $0 / 2$ & $2 / 2$ & $1 / 1$ & $1 / 1$ & $2 / 2$ & $0 / 2$ & - & $3 / 3$ & $4 / 4$ & - & - & $0 / 4(0)$ \\
\hline Pasteurella pneumotropica & - & $1 / 1$ & - & $1 / 1$ & - & - & - & - & - & - & - & $0 / 1$ & - & - & $1 / 1$ & $1 / 1$ & - & $1 / 1$ & $0 / 1$ & $1 / 1$ & - & - & $0 / 1(0)$ \\
\hline Pasteurella species & - & $1 / 1$ & - & $2 / 2$ & - & - & - & - & - & - & - & $1 / 1$ & - & - & $0 / 1$ & $1 / 1$ & - & - & $1 / 1$ & $1 / 1$ & - & - & $0 / 1(0)$ \\
\hline Proteus mirabillis & - & $3 / 3$ & - & $1 / 1$ & $1 / 1$ & - & $2 / 2$ & $0 / 1$ & $1 / 1$ & $2 / 2$ & $1 / 2$ & - & - & - & $1 / 3$ & $0 / 1$ & - & $1 / 3$ & $1 / 2$ & $2 / 2$ & - & - & $2 / 3(66.7)$ \\
\hline Providencia stuartii & $1 / 1$ & - & - & - & $1 / 1$ & - & $0 / 1$ & - & $1 / 1$ & $1 / 1$ & - & $1 / 1$ & - & - & - & $0 / 1$ & - & - & $1 / 1$ & - & - & - & $0 / 1(0)$ \\
\hline Pseudomonas aeruginosa & $2 / 2$ & $2 / 3$ & - & $1 / 1$ & $2 / 2$ & $1 / 1$ & $0 / 2$ & $1 / 2$ & $2 / 2$ & $2 / 3$ & $1 / 2$ & $0 / 1$ & $0 / 2$ & $1 / 1$ & $0 / 1$ & $0 / 1$ & $0 / 1$ & - & $1 / 2$ & $1 / 1$ & - & $1 / 2$ & $2 / 4(50)$ \\
\hline Pseudomonas species & - & $0 / 1$ & - & $0 / 1$ & $1 / 1$ & - & $0 / 2$ & $0 / 2$ & $1 / 2$ & $0 / 2$ & $0 / 1$ & $0 / 1$ & $1 / 2$ & - & - & - & $0 / 1$ & - & $1 / 2$ & $1 / 1$ & - & $1 / 1$ & $2 / 2(100)$ \\
\hline Serratia marcescens & - & $1 / 1$ & - & - & $1 / 1$ & - & $0 / 1$ & - & $1 / 1$ & $1 / 1$ & - & $1 / 1$ & - & - & - & $0 / 1$ & - & - & $1 / 1$ & - & - & - & $0 / 1(0)$ \\
\hline Staphylococci aureus & $2 / 2$ & $1 / 2$ & - & $2 / 2$ & - & - & $1 / 2$ & - & $1 / 1$ & $1 / 2$ & - & $1 / 2$ & $0 / 1$ & - & $2 / 3$ & $1 / 1$ & $1 / 1$ & $1 / 2$ & $0 / 4$ & $3 / 3$ & - & - & $1 / 4(25)$ \\
\hline Staphylococcus hyicus & $1 / 1$ & $3 / 3$ & - & $1 / 1$ & - & - & $3 / 3$ & - & - & - & $1 / 1$ & - & - & - & $2 / 3$ & $1 / 2$ & $2 / 3$ & - & $0 / 3$ & $2 / 2$ & - & - & $1 / 3(33.3)$ \\
\hline Staphylococci - coagulase negative & $6 / 6$ & $8 / 9$ & $1 / 1$ & $3 / 3$ & $4 / 4$ & $1 / 1$ & $8 / 11$ & $2 / 4$ & $4 / 4$ & $4 / 4$ & $5 / 5$ & $4 / 6$ & $5 / 6$ & $1 / 1$ & $2 / 4$ & - & $3 / 8$ & $0 / 2$ & $6 / 12$ & $7 / 7$ & - & $2 / 2$ & $4 / 15(35.7)$ \\
\hline Streptococci - alpha haemolytic & $1 / 1$ & $3 / 5$ & - & $1 / 2$ & $2 / 2$ & $1 / 2$ & $0 / 3$ & $0 / 1$ & $1 / 2$ & $0 / 1$ & $1 / 1$ & $1 / 2$ & $1 / 1$ & - & $1 / 4$ & $1 / 2$ & $1 / 1$ & $0 / 3$ & $1 / 5$ & $2 / 3$ & - & - & $4 / 6(66.7)$ \\
\hline Streptococci - beta haemolytic & $1 / 2$ & $1 / 1$ & - & $1 / 2$ & - & - & $0 / 2$ & - & $1 / 1$ & - & - & $0 / 2$ & $2 / 3$ & - & - & - & - & $0 / 1$ & $1 / 3$ & $2 / 3$ & - & - & $3 / 4(75)$ \\
\hline Streptococci-non-haemolytic & $1 / 1$ & - & - & - & - & - & $0 / 1$ & - & - & - & - & $0 / 1$ & $0 / 1$ & - & - & - & - & - & $0 / 1$ & $1 / 1$ & - & - & $1 / 1(100)$ \\
\hline Streptococcus bovis & - & $1 / 1$ & - & - & - & - & - & - & & - & - & $1 / 1$ & - & - & $0 / 1$ & $0 / 1$ & - & $0 / 1$ & $1 / 1$ & $1 / 1$ & & - & $0 / 1(0)$ \\
\hline Streptococcus dysgalactiae & $2 / 2$ & $1 / 1$ & - & - & - & $2 / 2$ & $1 / 1$ & - & - & - & $1 / 1$ & - & - & - & $0 / 1$ & $1 / 2$ & $1 / 1$ & $1 / 2$ & $1 / 3$ & $3 / 3$ & - & $0 / 1$ & $2 / 3(66.7)$ \\
\hline Streptococcus Lancefield Group C & $1 / 1$ & $5 / 5$ & - & $2 / 2$ & $1 / 1$ & - & $2 / 3$ & - & - & $2 / 2$ & $1 / 2$ & $0 / 2$ & - & - & $3 / 5$ & $2 / 2$ & $1 / 2$ & $2 / 2$ & $2 / 5$ & $5 / 5$ & - & - & $1 / 5(20)$ \\
\hline
\end{tabular}


Table 3. Cont

\begin{tabular}{|c|c|c|c|c|c|c|c|c|c|c|c|c|c|c|c|c|c|c|c|c|c|c|c|}
\hline \multirow[b]{2}{*}{ Bacterial Species } & \multicolumn{22}{|c|}{ Antimicrobial Used For Testing Susceptibility $a, b$} & \multirow[b]{2}{*}{ MDR (\%) } \\
\hline & $\sum_{4}^{\swarrow}$ & $\sum_{4}^{U}$ & 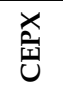 & $\sum_{\vec{T}}^{\mathbb{T}}$ & $\vec{y}$ & ôt & 茎 & $\frac{\pi}{4}$ & 总 & $\begin{array}{l}\text { O } \\
\text { 罗 }\end{array}$ & $\begin{array}{l}u \\
\text { und } \\
\text { in }\end{array}$ & 点 & $\underset{z}{z}$ & $\frac{\pi}{\Sigma}$ & $\underset{\widetilde{I}}{\overparen{I}}$ & $\Xi$ & $\vec{U}$ & $Z_{Z}$ & $\stackrel{\vec{y}}{\vec{F}}$ & $\sum_{i}^{\infty}$ & $\vec{\Xi}$ & 층 & \\
\hline Streptococcus Lancefield Group D & - & $1 / 1$ & $2 / 2$ & - & - & - & $0 / 1$ & $0 / 1$ & $1 / 1$ & $1 / 2$ & $1 / 1$ & - & $0 / 2$ & - & - & - & $0 / 1$ & - & $0 / 1$ & - & - & $0 / 1$ & $2 / 2(100)$ \\
\hline Streptococcus porcinus & - & $1 / 1$ & - & - & - & - & $1 / 1$ & - & 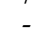 & - & - & - & - & - & - & $0 / 1$ & $0 / 1$ & - & $0 / 1$ & - & - & - & $1 / 1(100)$ \\
\hline Streptococci species & - & $2 / 3$ & - & $1 / 1$ & $1 / 2$ & - & $2 / 3$ & $0 / 1$ & $1 / 2$ & $1 / 2$ & - & $0 / 1$ & $1 / 1$ & - & $0 / 2$ & $1 / 1$ & $1 / 1$ & $0 / 1$ & $0 / 1$ & $1 / 1$ & - & - & $2 / 3(66.7)$ \\
\hline Streptococcus suis & - & $4 / 4$ & - & - & - & - & $1 / 2$ & $0 / 1$ & $1 / 1$ & $0 / 1$ & $1 / 1$ & $0 / 1$ & $0 / 1$ & - & $2 / 3$ & $1 / 3$ & $1 / 3$ & $0 / 1$ & $0 / 4$ & $1 / 2$ & - & $0 / 1$ & $3 / 4(75)$ \\
\hline Streptococcus viridans & - & $0 / 1$ & - & - & - & - & - & - & - & - & - & $1 / 1$ & - & - & $1 / 1$ & - & - & $0 / 1$ & $0 / 1$ & $1 / 1$ & - & - & $0 / 1(0)$ \\
\hline Trueperella pyogenes & $1 / 1$ & $1 / 1$ & - & - & - & - & $0 / 1$ & $0 / 1$ & $0 / 1$ & $1 / 1$ & $0 / 1$ & - & $0 / 1$ & - & - & $0 / 1$ & $0 / 1$ & - & $0 / 1$ & - & $0 / 1$ & $1 / 1$ & $1 / 1(100)$ \\
\hline Unidentified bacterial isolates & $4 / 4$ & $2 / 4$ & - & - & $1 / 1$ & - & $1 / 3$ & $0 / 1$ & $0 / 1$ & $1 / 2$ & $0 / 1$ & $0 / 1$ & $3 / 4$ & - & $1 / 2$ & t & $2 / 2$ & $0 / 1$ & $4 / 5$ & $5 / 5$ & - & $1 / 1$ & $2 / 6(33.3)$ \\
\hline
\end{tabular}

a AMX = amoxicillin and clavulanic acid; AMC = ampicillin; CEPH = cephalothin; CEFT = ceftiofur; CEPX = cephalexin; OXA = oxacillin; PEN = penicillin; APR = aprmycin; $\mathrm{GEN}=$ gentamicin; NEO = neomycon; SPEC = spectinomycin; STR = streptomycin; ENR = enrofloxacin; MAR = marbofloxacin; ERY = erythromycin; TIL = tilmicosin; CLI = clindamycin; $\mathrm{LNC}=$ lincomycin; TET $=$ tetracycline; TMS $=$ trimethoprim-sulphonamide; $\mathrm{CHL}=$ chloramphenicol; POLY $=$ polymixin B. ${ }^{\mathrm{b}}$ Susceptibility to ceftazidime was reported for $E$. coli $(1 / 1$ susceptible) and two other unidentified isolates (2/2 susceptible) only; susceptibility to tylosin was reported for $E$. coli (0/1 susceptible) only; susceptibility to fusidic acid was reported for $S$. dysgalactiae (2/2 susceptible) only. ${ }^{c}$ Proportion and percentage of tested isolates of that species or genus (row) exhibiting multidrug resistance. 


\section{Discussion}

This study provides a broader overview of the bacterial species associated with infectious disease in New Zealand pigs than has previously been published $[4,16,17]$. The distribution of isolates was biased towards bacterial pathogens associated with enteric disease, respiratory disease, meningitis and sudden death $[15,23,24]$. Although the size of the study population was small, the percentage of isolates expressing MDR was generally higher than that recently reported for other farm animal species in New Zealand [25,26]. The limited number of sample submissions from this population is at odds with estimates of antimicrobial sales in the pork industry, indicating that, in common with other industry sectors, factors other than culture and susceptibility results are the main drivers of antimicrobial use in New Zealand $[4,27,28]$.

E. coli was the most common bacterial species identified within this population, but only a quarter of these isolates (141/186) were serotyped, and of these, half were positive for the K88 adhesin associated with postweaning diarrhoea in pigs [29]. Peer-reviewed publications describing the pathogenicity of porcine strains of E. coli in New Zealand are scant [4,30,31]. Effective vaccination may be used as one of the strategies to prevent porcine E. coli diarrhoea but requires serotyping to identify suitable vaccinate strains [29]. Evidence of subtyping in the database studied was limited, and there was no information on how the culture results were utilised by submitting veterinarians. It is recommended that isolates are routinely serotyped for differentiation of strains of differing pathogenicity in pigs, and to identify those of public health significance $[30,31]$.

A. pleuropneumoniae, the second most commonly cultured isolate, is widespread in New Zealand's pigs, and is an important cause of pleuropneumonia and septicaemia [3,13,32]. The current true prevalence in the pig population is unknown, but given the frequency of animal movement between large commercial and small-scale piggeries [10], it is possible that the $5.5 \%$ of isolates is associated with an increase in prevalence from $2.7 \%$ of porcine abattoir samples found in 1998 [3,13]. The isolation of S. suis from New Zealand pigs was first described in the 1980s and is associated with neurologic and arthritic disease [33]. In addition to its importance as a pig pathogen, the organism is a zoonosis of emerging importance, with farmers, food processors and veterinarians at risk [34,35]. A high prevalence of serotypes 1 and 2 was found in the New Zealand pig population in the 1980s, but there are no contemporary published data [33]. In the current data set, most isolates (95.3\%) were not typed. However, serotyping is critical in understanding the epidemiology and pathogenesis of the $S$. suis in pigs, as well as its pathogenicity as a zoonotic agent $[35,36]$.

Campylobacter spp. were also frequently isolated. C. coli is considered the dominant species in pigs [24], but in the current study, C. jejuni isolates were more common. However, most Campylobacter spp. isolates (55.9\%) were not speciated, limiting further conclusions. Similar numbers of each species were isolated in a study of pig offal in New Zealand [17], but C. jejuni is considered to be of greater public health significance [37,38]. Salmonella spp. and Yersinia spp. were infrequently isolated. Although pigs are routinely targeted for surveillance to detect these organisms at abattoirs [18], the low numbers of Salmonella spp. isolates in the current study are consistent with low numbers in recent governmental animal health surveillance reports for pigs [39].

Although fewer than $20 \%$ of isolates from $13 \%$ of submissions were tested for antimicrobial susceptibility, these rates are markedly higher than those reported in contemporary New Zealand studies of beef and preproduction cattle (9.2\% and $6.6 \%$ respectively), and sheep (5.3\% and $2.5 \%$ respectively) [26]. A recent report in the New Zealand dairy industry showed that $21 \%$ of veterinarians utilise susceptibility testing, but it is unclear if this corresponds with the number of submissions for bacterial culture [28]. The low level of susceptibility testing is concerning as it further emphasises a disparity between susceptibility-guided prudent antimicrobial use in the livestock industries in New Zealand and annual antimicrobials sales [4,25,27]. Evidence for New Zealand livestock veterinarians is scant, but a recent survey found that $80 \%$ of dairy veterinarians prescribe antimicrobials based on a diagnosis, and $65 \%$ as a test for response to therapy, in lieu of bacterial culture and antimicrobial susceptibility testing [28]. For some pathogenic organisms, the identification of species may direct 
other control measures, such as vaccination (e.g., E. coli) or providing zinc oxide in feed at weaning [40], rather than antimicrobial use [23,29]. Given the low submission rate for pigs and lack information on how these laboratory results are utilised, closer scrutiny of the drivers for sample submission and antimicrobial use is recommended.

Interpretation of the significance of antimicrobial susceptibility results is limited by the small number of isolates within each species or genus grouping tested, and the use of different antimicrobial panels for the same bacterial species in the dataset (Table 2). For E. coli isolates, the most common isolate tested, susceptibility to tetracycline (25\%), was low, but high to neomycin (92\%), enrofloxacin (92\%), and trimethoprim-sulphonamide (95\%). These findings are largely in agreement with a United Kingdom study of 152 isolates that comparable susceptibilities for tetracycline (20\%), neomycin (95\%) and enrofloxacin (80-93\%), but there was a better response to trimethoprim-sulphonamide (55\%) [40]. A larger pool of E. coli clinical isolates $(n=2144)$ from the United States had a low susceptibility to tetracycline and neomycin (50\%), a strong response to enrofloxacin (98\%), and moderate susceptibility to trimethoprim-sulphonamide (74\%) [40]. A 2001 study of New Zealand 296 E. coli isolates from conventionally farmed healthy pigs identified a higher susceptibility to tetracycline (60\%), and a similar response to neomycin (99\%) (4). Susceptibility to enrofloxacin and trimethoprim-sulphonamide were not tested by Nulsen et al. (2008), but susceptibility to amoxicillin in the current group of submissions (60\%) was markedly lower than that previously determined for healthy New Zealand pigs (98.3\%) [4].

Antimicrobials are an important tool in combating the bacterial pathogens associated with Porcine Respiratory Disease Complex (PRDC) [41]. A. pleuropneumoniae and P. multocida are common bacterial pathogens associated with PRDC, and were common isolates cultured in the current study. None of the isolates from these two bacterial species displayed MDR, in agreement with a recent study of these pathogens in Spain [41]. However few isolates within the current database were subjected to susceptibility testing.

As described for other livestock species in New Zealand, susceptibility and MDR data for Campylobacter spp., Salmonella spp., and Yersinia spp. were not available [25]. Campylobacter coli and C. jejuni isolates from pig offal sources within some regions of New Zealand have a higher level of resistance to erythromycin (35.7\%) than other livestock, or human-derived isolates [17]. Campylobacter spp. are the second most common zoonoses of public health concern in New Zealand [42], and the lack of AMR for the porcine isolates in this database limits the value of these data for informing prudent use guidelines in the pork industry. The other most common isolates, A. pleuropneumoniae, and S. suis had so few susceptibility data that meaningful recommendations for antimicrobial use cannot be made.

The total level of MDR (42.6\%) was double that reported for a contemporary studies of AMR and MDR in New Zealand beef and preproduction dairy cattle (20.7\%) and sheep (20.5\%), and 60\% greater than the rate for mixed bacterial infections in New Zealand equine neonates $(26 \%)[25,26,43]$. Although antimicrobial use practices were not documented in the current study, there is evidence that the oral administration of antimicrobials, as commonly practiced within the pork industry, may be a significant risk factor for AMR and MDR for bacteria recovered from pigs [5]. For individual bacterial species, meaningful comparisons the published literature are limited by small sample size in the current study. However, MDR rates for the more commonly evaluated bacterial species of E. coli and coagulase-negative Streptococci were moderate.

These data provide an overview of the different bacterial species associated with diseased New Zealand pigs, but in common with recent works in other species, the lack of clinical context driving submission behaviours and the size of the data set limit the broader application of these findings [25,43,44]. Inconsistent and incomplete signalment data and nonstandardised approaches to susceptibility testing during the period of review prevented the use of epidemiologic modelling to identify risk factors for AMR. Accurate signalment information, culture, and antimicrobial susceptibility results underpin meaningful laboratory interpretation, and effective AMR surveillance $[45,46]$. The applicability of the current findings across the New Zealand pork industry is limited due to the low numbers of isolates submitted for antimicrobial susceptibility testing. Furthermore, the frequency of AMR and 
MDR must be interpreted with caution. Antimicrobial panels used for susceptibility testing did not differentiate between intrinsically resistant isolates and those expressing acquired resistance [47]. For example, Enterobacteriaceae phenotypes include those intrinsically resistant to benzylpenicillin, macrolides, lincosamides, streptogramins, and rifampin [47]. Ideally, antimicrobial panels used for susceptibility testing should be specifically selected for the bacterial isolate tested, using animal-specific CSLI resistance breakpoints [48].

\section{Conclusions}

This is the first report on the breadth of bacterial species, antimicrobial susceptibility and MDR based on a wide spectrum of isolates from laboratory submissions for New Zealand pigs. MDR rates are higher in pigs and those recently reported for other New Zealand livestock species. These findings contribute to an understanding of the scope of bacterial species associated with porcine disease in New Zealand but provide limited information on AMR in this population. The use of bacterial culture and susceptibility testing in the New Zealand pork industry is limited. More data on sample submission behaviours, antimicrobial drug uses in the pork industry, and the susceptibilities of important porcine bacterial isolates are required to inform guidelines for their prudent use in this industry. Data captured by the organised monitoring of bacterial isolates of note or potential public health importance from pigs may inform strategies that reduce their prevalence and transmission of those with zoonotic potential in New Zealand.

Author Contributions: Conceptualization, C.B.R. and K.L.C.; methodology, C.B.R.; formal analysis, C.B.R., J.P.B. and K.E.L.; writing - original draft preparation, C.B.R.; writing-review and editing, C.B.R., K.E.L., K.L.C., J.P.B., and E.G.; visualization, C.B.R. and J.P.B. All authors have read and agreed to the published version of the manuscript.

Funding: This research received no external funding.

Conflicts of Interest: The authors declare no conflict of interest.

\section{References}

1. Abatih, E.N.; Ersbøll, A.K.; Lo Fo Wong, D.M.A.; Emborg, H.D. Space-time clustering of ampicillin resistant Escherichia coli isolated from Danish pigs at slaughter between 1997 and 2005. Prev. Vet. Med. 2009, 89, 90-101. [CrossRef]

2. Heffernan, H.; Wong, T.L.; Lindsay, J.; Bowen, B.; Woodhouse, R. A Baseline Survey of Antimicrobial Resistance in Bacteria from Selected New Zealand Foods; 2009-2010 MAF Technical Paper No: 2011/53; Ministry of Agriculture and Forestry: Christchurch, New Zealand, 2011.

3. Stärk, K.D.C.; Pfeiffer, D.U.; Morris, R.S. Risk factors for respiratory diseases in New Zealand pig herds. New Zealand Vet. J. 1998, 46, 3-10. [CrossRef]

4. Nulsen, M.F.; Mor, M.B.; Lawton, D.E.B. Antibiotic resistance among indicator bacteria isolated from healthy pigs in New Zealand. N. Z. Vet. J. 2008, 56, 29-35. [CrossRef] [PubMed]

5. Burow, E.; Simoneit, C.; Tenhagen, B.-A.; Käsbohrer, A. Oral antimicrobials increase antimicrobial resistance in porcine E. coli-A systematic review. Prev Vet. Med. 2014, 113, 364-375. [CrossRef] [PubMed]

6. Österberg, J.; Wingstrand, A.; Nygaard Jensen, A.; Kerouanton, A.; Cibin, V.; Barco, L.; Denis, M.; Aabo, S.; Bengtsson, B. Antibiotic resistance in Escherichia coli from pigs in organic and conventional farming in four European countries. PLoS ONE 2016, 11, e0157049. [CrossRef] [PubMed]

7. Arndt, E.R.; Farzan, A.; MacInnes, J.I.; Friendship, R.M. Antimicrobial resistance of Streptococcus suis isolates recovered from clinically ill nursery pigs and from healthy pigs at different stages of production. Can. Vet. J. 2019, 60, 519-522. [PubMed]

8. Morel, P.C.H. Pig production. In Livestock Production in New Zealand; Stafford, K., Ed.; Massey University Press: Auckland, New Zealand, 2017; pp. 172-213.

9. Infoshare, Statistics New Zealand. Available online: www.stats.govt.nz/infoshare (accessed on 12 June 2019). 
10. Neumann, E.J.; Pearson, A.B.; Sanson, R.L.; Nicoll, K.J.; Clement, F.L. The frequency and distance of movements of pigs and semen between commercial and non-commercial piggeries in New Zealand. N. Z. Vet. J. 2013, 61, 77-86. [CrossRef]

11. Gongora, J.; Garkavenko, O.; Moran, C. Origins of Kune Kune and Auckland Island pigs in New Zealand. In Proceedings of the. 7th World Congress on Genetics Applied to Livestock Production, Montpellier, France, 19-23 August 2002; Volume 26, pp. 26-39.

12. Christensen, N.H.; Cullinane, L.C. Monitoring the health of pigs in New Zealand abattoirs. N. Z. Vet. J. 1990, 38, 136-141. [CrossRef]

13. Hilbink, F.; de Lisle, G.W.; Penrose, M.; Aldersley, B.; Carman, M. Actinobacillus pleuropneumoniae in New Zealand pigs. N. Z. Vet. J. 1992, 40, 173-175. [CrossRef]

14. Bolt, I.; Marshall, R. The epidemiology of Leptospira interrogans serovar Pomona in grower pig herds. N. Z. Vet. J. 1995, 43, 10-15. [CrossRef]

15. Jamaludin, R.; Blackall, P.J.; Hansen, M.F.; Humphrey, S.; Styles, M. Phenotypic and genotypic characterisation of Pasteurella multocida isolated from pigs at slaughter in New Zealand. N. Z. Vet. J. 2005, 53, 203-207. [CrossRef] [PubMed]

16. Hodges, R.T.; Young, G.W. Prevalence and in-vitro antimicrobial sensitivity of Bordetella bronchiseptica in the nasal cavity of pigs. N. Z. Vet. J. 1984, 32, 111-114. [CrossRef] [PubMed]

17. Harrow, S.A.; Gilpin, B.J.; Klena, J.D. Characterization of erythromycin resistance in Campylobacter coli and Campylobacter jejuni isolated from pig offal in New Zealand. J. Appl. Microbiol. 2004, 97, 141-148. [CrossRef] [PubMed]

18. Lal, A.; Baker, M.G.; French, N.P.; Dufour, M.; Hales, S. The epidemiology of human salmonellosis in New Zealand 1997-2008. Epidemiol. Infect. 2012, 140, 1685-1694. [CrossRef] [PubMed]

19. Baker, M.G.; Thornley, C.N.; Lopez, L.D.; Garrett, N.K.; Nicol, C.M. A recurring salmonellosis epidemic in New Zealand linked to contact with sheep. Epidemiol. Infect. 2007, 135, 76-83. [CrossRef]

20. Jordan, D. Surveillance for antibiotic resistant Escherichia coli in food animals. Commun. Dis. Intell 2003, 27, S117-S120.

21. Cockerill, F.R.; Wikler, M.A.; Alder, J.; Dudley, M.N.; Eliopoulos, G.M.; Ferraro, M.J.; Hardy, D.J.; Hecht, D.W.; Hindler, J.A.; Patel, J.B.; et al. Performance Standards for Antimicrobial Disk Susceptibility Testing: Approved Standard National Committee for Clinical Laboratory Standards; Institute CLS: Wayne, NJ, USA, 2012.

22. Magiorakos, A.-P.; Srinivasan, A.; Carey, R.B.; Carmeli, Y.; Falagas, M.E.; Giske, C.G.; Harbarth, S.; Hindler, J.F.; Kahlmeter, G.; Olsson-Liljequist, B.; et al. Multidrug-resistant, extensively drug-resistant and pandrug-resistant bacteria: An international expert proposal for interim standard definitions for acquired resistance. Clin. Microbiol. Infect. 2012, 18, 268-281. [CrossRef]

23. Wills, R.W. Diarrhea in growing-finishing swine. Vet. Clin. N. Am. Food Anim. Pr. 2000, 16, $135-161$. [CrossRef]

24. Burch, D.G.S.; Duran, C.O.; Aerestrup, F.M. Guidelines for antimicrobial use in swine. In Guide to Antimicrobial Use in Animals; Guardabassi, L., Jensen, L.B., Kruse, H., Eds.; Blackwell Publishing Ltd.: Oxford, UK, 2008; pp. 102-125.

25. Lawrence, K.E.; Wakeford, L.; Tooms-Ruane, L.J.; MacLachlan, C.; Pfeffer, H.; Gibson, I.R.; Benschop, J.; Riley, C.B. Bacterial isolates, antimicrobial susceptibility and multidrug resistance in cultures from samples collected from beef and pre-production dairy cattle in New Zealand (2003-2016). N. Z. Vet. J. 2019, 67, 180-187. [CrossRef]

26. Riley, C.B.; Pfeffer, H.; MacLachlan, C.; Wakeford, L.; Gibson, I.R.; Benschop, J.; Lawrence, K.E. Isolates, antimicrobial susceptibility profiles and multidrug resistance of bacteria cultured from samples collected from sheep in New Zealand (2003-2016). N. Z. Vet. J. 2020. [CrossRef]

27. Hillerton, J.E.; Irvine, C.R.; Bryan, M.A.; Scott, D.; Merchant, S.C. Use of antimicrobials for animals in New Zealand, and in comparison with other countries. N. Z. Vet. J. 2017, 65, 71-77. [CrossRef] [PubMed]

28. McDougall, S.; Compton, C.W.R.; Botha, N. Factors influencing antimicrobial prescribing by veterinarians and usage by dairy farmers in New Zealand. N. Z. Vet. J. 2017, 65, 84-92. [CrossRef] [PubMed]

29. Fairbrother, J.M.; Nadeau, E.; Gyles, C.L. Escherichia coli in postweaning diarrhea in pigs: An update on bacterial types, pathogenesis, and prevention strategies. Anim. Health Res. Rev. 2005, 6, 17-39. [CrossRef] 
30. Brooks, H.J.L.; Mollison, B.D.; Bettelhelm, K.A.; Matejka, K.; Paterson, K.A.; Ward, V.K. Occurrence and virulence factors of non-O157 Shiga toxin-producing Escherichia coli in retail meat in Dunedin, New Zealand. Lett. Appl. Microbiol. 2001, 32, 118-122. [CrossRef]

31. Wong, T.L.; MacDiarmid, S.; Cook, R. Salmonella, Escherichia coli O157:H7 and E. coli biotype 1 in a pilot survey of imported and New Zealand pig meats. Food Microbiol. 2009, 26, 177-182. [CrossRef]

32. Baskerville, A. Pneumonia of pigs: A review. N. Z. Vet. J. 1981, 29, 216-218. [CrossRef]

33. Roberston, I.D.; Blackmore, D.K. Prevalence of Streptococcus-suis type-1 and type-2 in domestic pigs in Australia and New Zealand. Vet. Rec. 1989, 124, 391-394.

34. Robertson, I.D.; Blackmore, D.K. Occupational exposure to Streptococcus suis type 2. Epidemiol. Infect. 1989, 103, 157-164. [CrossRef]

35. Goyette-Desjardins, G.; Auger, J.-P.; Xu, J.; Segura, M.; Gottschalk, M. Streptococcus suis, an important pig pathogen and emerging zoonotic agent-An update on the worldwide distribution based on serotyping and sequence typing. Emerg. Microbes. Infect. 2014, 3, e45. [CrossRef]

36. Prüfer, T.L.; Rohde, J.; Verspohl, J.; Rohde, M.; de Greeff, A.; Willenborg, J.; Valentin-Weigand, P. Molecular typing of Streptococcus suis strains isolated from diseased and healthy pigs between 1996-2016. PLoS ONE 2019, 14, e0210801. [CrossRef]

37. Moore, J.E.; Barton, M.D.; Blair, I.S.; Corcoran, D.; Dooley, J.S.G.; Fanning, S.; Kempf, I.; Lastovica, A.J.; Lowery, C.L.; Matsuda, M.; et al. The epidemiology of antibiotic resistance in Campylobacter. Microbes. Infect. 2006, 8, 1955-1966. [CrossRef] [PubMed]

38. Lim, E.; Lopez, L.; Cressey, P.; Pirie, R. Foodborne Disease in New Zealand 2010; Client Report FW 11031; Institute of Environmental Science and Research Ltd.: Christchurch, New Zealand, 2015.

39. Watts, J.; Jaramillo, D.; Walker, K. Animal health surveillance. Surveillance 2017, 44, 13-17.

40. Burch, D.G.S. Antimicrobial drug use in swine. In Antimicrobial Therapy in Veterinary Medicine, 5th ed.; Giguère, S., Prescott, J.F., Dowling, P.M., Eds.; John Wiley \& Sons Inc.: Ames, IA, USA, 2013; pp. 553-568.

41. Vilaró, A.; Novell, E.; Enrique-Tarancón, V.; Balielles, J.; Vilalta, C.; Martinez, S.; Sauce, L.J.F. Antimicrobial susceptibility pattern of poor sign respiratory bacteria in Spain. Antibiotics 2020, 9, 402. [CrossRef] [PubMed]

42. Wong, T.L.; Hollis, L.; Cornelius, A.; Nicol, C.; Cook, R.; Hudson, J.A. Prevalence, numbers and subtypes of Campylobacter jejuni and Campylobacter coli in uncooked retail meat samples. J. Food Prot. 2007, 70, 566-573. [CrossRef]

43. Toombs-Ruane, L.; Riley, C.B.; Kendall, A.T.; Hill, K.E.; Benschop, J.; Rosanowski, S.M. Antimicrobial susceptibility of bacteria isolated from neonatal foal samples submitted to a New Zealand veterinary pathology laboratory (2004 to 2013). N. Z. Vet. J. 2016, 64, 107-111. [CrossRef]

44. Petrovski, K.R.; Laven, R.A.; Lopez-Villalobos, N. A descriptive analysis of the antimicrobial susceptibility of mastitis-causing bacteria isolated from samples submitted to commercial diagnostic laboratories in New Zealand (2003-2006). N. Z. Vet. J. 2011, 59, 59-66. [CrossRef]

45. Hodgson, J.L.; Hughes, K.J.; Hodgson, D.R. Diagnosis of bacterial infections. Part 2: Bacterial cultivation, susceptibility testing and interpretation. Equine. Vet. Educ. 2008, 20, 658-666. [CrossRef]

46. Schwarz, S.; Silley, P.; Simjee, S.; Woodford, N.; van Duijkeren, E.; Johnson, A.P.; Gaastra, W. Assessing the antimicrobial susceptibility of bacteria obtained from animals. Vet. Microbiol. 2010, 141, 1-4. [CrossRef]

47. Boerlin, P.; White, D.G. Antimicrobial resistance and its epidemiology. In Antimicrobial Therapy in Veterinary Medicine, 5th ed.; Giguère, S., Prescott, J.F., Dowling, P.M., Eds.; John Wiley \& Sons Inc.: Ames, IA, USA, 2013; pp. 21-40.

48. Rubin, J.E. Antimicrobial susceptibility testing methods and interpretation of results. In Antimicrobial Therapy in Veterinary Medicine, 5th ed.; Giguère, S., Prescott, J.F., Dowling, P.M., Eds.; John Wiley \& Sons Inc.: Ames, IA, USA, 2013; pp. 11-20.

(C) 2020 by the authors. Licensee MDPI, Basel, Switzerland. This article is an open access article distributed under the terms and conditions of the Creative Commons Attribution (CC BY) license (http://creativecommons.org/licenses/by/4.0/). 